\title{
Visuospatial attention: the role of target contrast and task difficulty when assessing the effects of cues
}

Robert J Snowden, Jennifer Willey\#, Janice L Muir

School of Psychology, Cardiff University, PO Box 901, Cardiff CF1 3YG, Wales, UK;

e-mail: snowden@cardiff.ac.uk; \# also at Caswell Clinic, Glanrhyd Hospital, Bridgend, Mid Glamorgan CF31 4LN, Wales, UK

Received 14 April 2000, in revised form 30 November 2000

\begin{abstract}
Cueing paradigms have become popular in assessing the processes of attention. In two experiments we manipulated (i) the contrast of the target, and (ii) the similarity between the targets discriminated. We used a cue that would isolate the exogenous component of attention. Both a reduction in target contrast and an increase in target similarity raised overall reaction times by a similar amount; however, the target contrast manipulation produced a much greater cueing effect compared with the target similarity manipulation. The results suggest that manipulation of target contrast changes the attention cueing effect at a stage of attracting attention to a location of the target (the 'move' stage), rather than at a later processing stage.
\end{abstract}

\section{Introduction}

A popular paradigm for the investigation of visual attention has been the spatial pre-cueing paradigm (Posner et al 1980). Here a target is presented at one of two (or more) possible locations whilst the subject fixates at a central marker. In advance of the target, a 'cue' to one of these locations is presented. This pre-cue can be either valid (the target occurs at the location suggested by the cue) or invalid (the target occurs at another location). Responses on valid trials are typically faster than on invalid trials, which we term the 'cue validity' effect.

The spatial pre-cueing paradigm has been examined in many populations. It has been applied to normal ageing (Nissen and Corkin 1985; Tellinghuisen et al 1996), development in childhood (Enns and Brodeur 1989), Alzheimer's disease (Parasuraman et al 1992), Parkinson's disease (eg Bennett et al 1995), schizophrenia (Maruff and Pantelis 1999; Willey et al, submitted), brain damage in humans (Posner et al 1984; Rafal and Robertson 1995), and experimental brain lesions in non-human primates (Posner and Raichle 1997). In many of these conditions attentional processes appear to be disrupted. As an example of such research we shall examine the finding that reaction times to invalid trials are disproportionately slowed compared with neutral or valid trials in older subjects (Nissen and Corkin 1985).

The finding of such attentional deficits leaves open the question what causes this change. The task faced by the participant on each trial can be conceptualised as having two stages. First, the participants must move their attention to the target, and, second, they must process the target. The mechanisms involved in the first stage have been well explored. They have been broken down into three components involved in disengaging attention from its current location (thought to involve the posterior parietal cortex), moving attention to a new location (thought to involve the superior colliculus), and engaging it at the new location (thought to involve the pulvinar-for a review see Posner and Petersen 1990). Deficits in processing at this stage could indeed cause the pattern of results described above for old age. For example, if the participants had problems disengaging their attention, this would indeed lead to a disproportionate slowing for invalid trials (Posner et al 1984). The second stage involves extracting information from the target once attention has been engaged. Relatively little research 
has been carried out to examine this stage. However, deficits in the rate of extracting information could also produce the disproportionate loss for invalid trials. If attention is regarded as a resource that is unevenly split between the two possible target locations (Downing 1988; LaBerge and Brown 1989; Bundesen 1990; Bonnel et al 1992), then information is extracted more rapidly from the cued than the uncued location. If, in old age, the overall amount of resources is diminished, and the same amount of resources is still allocated to the valid location, then there will be less resources at the invalid location, and hence a disproportionate loss for targets at this location. Hence cueing effects could occur owing to changes in the 'move' stage, or in the 'process' stage.

Target luminance has been used as a tool to examine whereabouts cueing effects occur. Using additive factors logic, Hughes (1984) argued that, if luminance and cue validity interact, they must share a common stage of processing. As luminance or contrast $^{(1)}$ modulates the response of neurons early in visual processing, this would in turn suggest an 'early' site for the effects of cue validity. Hughes found no such interaction and thus argued that cue validity did not share a common stage of processing with contrast, which in turn argues for a 'late' site for the effects of cueing. Subsequently, Hawkins et al (1988) noted that Hughes had blocked the trials for the low-contrast and high-contrast targets separately. This allowed subjects the possibility of differing strategies for the two types of target. Hawkins et al therefore interleaved their targets of high and low contrast and now found an interaction between contrast and cue validity. However, it should also be noted that a further study in which target contrast was also manipulated and trials were interleaved did not reveal any interaction (Tellinghuisen et al 1996). Hence at this point it is unclear whether contrast does interact with cue validity. It should also be noted that in these three previous studies of the effects of target contrast upon cue validity central cues were used that were predictive of the target's location. This type of cue is normally termed an endogenous cue (Theeuwes 1994). It is often contrasted with a cue that automatically attracts attention to itself even though it is not predictive of target location-an exogenous cue. It appears that these two cues may have independent effects (Riggio and Kirsner 1997) and may use somewhat independent brain areas (Corbetta et al 1993). Thus the present study is the first in which the effects of target contrast for purely exogenous cueing have been examined.

If cue validity does interact with the contrast of the target, does this imply an 'early' site for attention? It is clear that contrast has an effect on the early stages of visual processing (Shapley 1986), but that the effects of target contrast propagate well into the visual system (Sclar et al 1990). Hence changes in contrast could affect both the 'move' stage and the 'process' stage of the cueing task. For example, reduction in target contrast would mean that targets occurring at the invalid location may be less able to summon attention to their location and hence a greater cueing effect would occur. Likewise, reduced contrast would mean that the stimulus would have to be processed longer in order to extract the necessary information for the decision process. Thus, by itself, contrast does not give much insight into the site at which attentional effects occur, as both stages would predict an interaction (thus making the negative results discussed above somewhat surprising).

In this paper we had therefore two aims. First, given the ambiguous pattern of results so far reported (Hughes 1984; Hawkins et al 1988; Tellinghuisen et al 1996), to re-examine the effects of target contrast upon cueing effects. We predict that as target contrast is reduced (i) reaction times (RTs) will increase, and (ii) the cue effect (invalid RT-valid RT) will increase. Second, we attempt to further localise the site of cueing

(1) The term luminance has generally been used in most of this literature. However, in vision science it is common to distinguish between the luminance (the overall light level that governs the state of adaptation) and the contrast of the target. We are interested in target contrast irrespective of luminance and therefore use contrast even for those studies that may have confounded the two. 
effects by manipulating the task difficulty independently of target contrast (see figure 1). The task of our participants was to identify which of two targets had been presented. The targets were a single line at some particular orientation, and task difficulty could be altered by varying the angle between the two possible targets (see figure 1). Such a manipulation should not affect the 'move' stage of attention (as the manipulation does not alter the salience of the target) but does alter the amount of information the observer must extract in order to identify the target (the 'process' stage). Therefore, if cueing effects occur at the 'process' stage, the manipulation of task difficulty should interact with cue validity. If no such effects occur, this would point to target contrast having its effects (if they exist) at the 'move' stage.

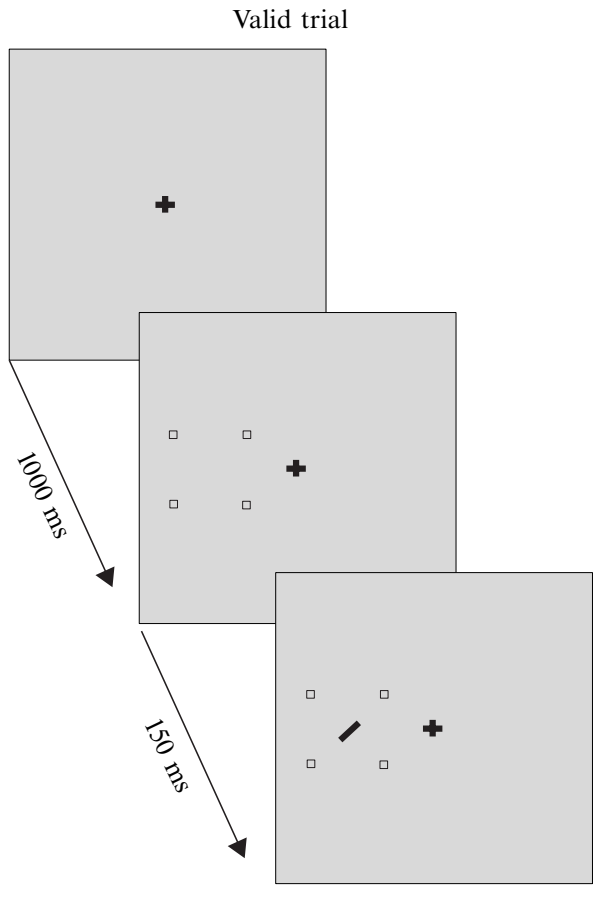

Experiment 1

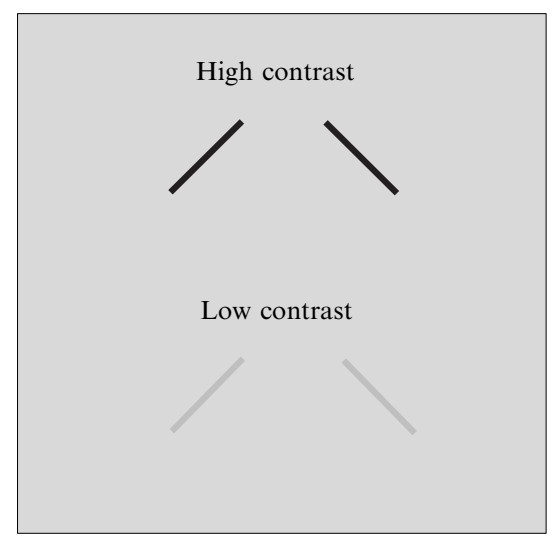

Invalid trial

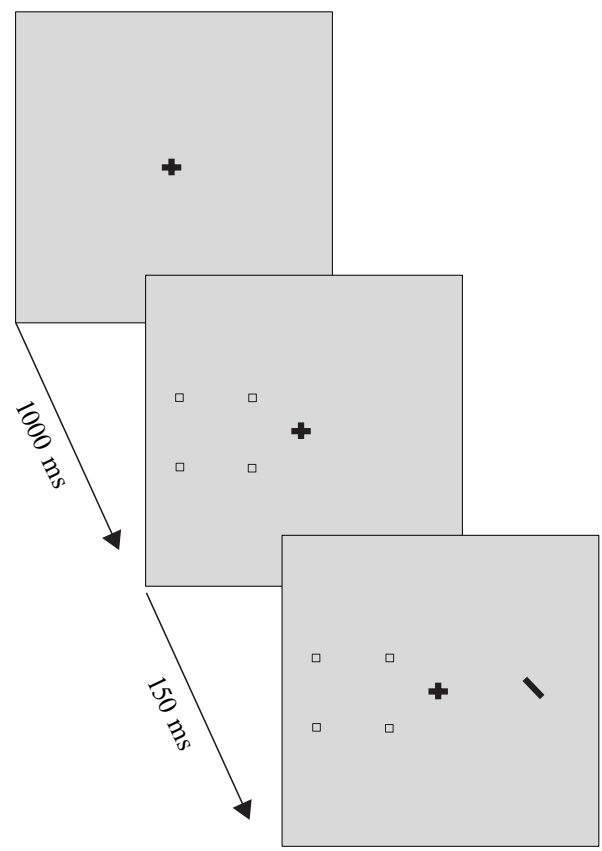

Experiment 2

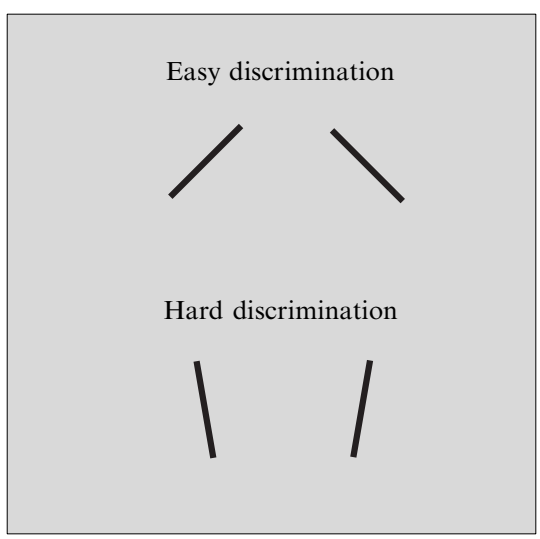

Figure 1. Upper part: the sequence of events for a valid and for an invalid trial. Lower part: a schematic example of the stimuli used in experiment 1 and in experiment 2 . 


\section{Experiment 1: Effect of target contrast}

In this experiment the tilt of the target line from vertical was large, making the discrimination task easy; however, the targets could appear at high or low contrast. Purely exogenous cueing (Theeuwes 1994) was achieved by having a cue occurring at a peripheral location that was not predictive of the location of the forthcoming target. A cue-target lead time of $150 \mathrm{~ms}$ was chosen to help optimise the exogenous component of attention (Müller and Rabbit 1989).

\subsection{Methods and materials}

2.1.1 Participants. Fifty participants (twelve males, thirty-eight females) were drawn from undergraduates at Cardiff University. The ages ranged from 18 to 46 years, with a mean age of 20.2 years. All were naïve as to the aims of the experiment and had not participated in similar experiments beforehand. No formal tests were made of visual acuity, but participants wore corrective lenses if they required them for reading. All volunteered for the experiment and were not paid for their time.

2.1.2 Apparatus. All stimuli were generated off-line with a graphics package (MacDraw Pro) and were displayed on the monitor $(26 \mathrm{~cm} \times 20 \mathrm{~cm})$ of a Mac LC computer. Stimulus presentations and reaction time recording were all controlled by this computer by means of the SuperLab software. The observer was seated with the eyes $57 \mathrm{~cm}$ from the screen in a dimly lit room. The head was not restrained. Eye movements and fixation were monitored via a camera (VISTA NCD 380 monocamera) mounted on top of the presentation screen, $57 \mathrm{~cm}$ from the eyes. The signal was sent to a TV monitor for visual inspection by the experimenter. No automated detection procedures were used.

2.1.3 Stimuli. The screen background level was a uniform white of luminance $50 \mathrm{~cd} \mathrm{~m}^{-2}$. The cues consisted of four small squares that defined a larger square. The small squares had a side of $0.5 \mathrm{deg}$ and defined a larger square of side $3.0 \mathrm{deg}$. This larger square was centred $7.5 \mathrm{deg}$ horizontally from the fixation cross (two $0.75 \mathrm{deg}$ lines). The cue elements were just the outline of the small squares. The lines that made up these outlines had a luminance of $2 \mathrm{~cd} \mathrm{~m}^{-2}$. The targets were lines of length $1.2 \mathrm{deg}$ and width $0.2 \mathrm{deg}$, and were dark relative to the background. The high-contrast targets had a luminance of $2 \mathrm{~cd} \mathrm{~m}^{-2}$ (Weber contrast $=\mathrm{d} L / L=48 / 50=0.96$ ), whereas the low-contrast targets had a luminance of $40 \mathrm{~cd} \mathrm{~m}^{-2}$ (Weber contrast $=\mathrm{d} L / L=$ $10 / 50=0.20$ ). The contrasts used were chosen after pilot experiments that confirmed that these contrasts produced a strong effect on reaction times whilst still being visible to the participants.

The targets were tilted at either $+45^{\circ}$ or $-45^{\circ}$. All targets were presented centred 7.5 deg horizontally displaced from the fixation point.

2.1.4 Procedure. The participants received a verbal description of the task to be performed. They were given explicit and correct information about the validity of the cues, and were asked to react to the presentation of the target as quickly as possible whist keeping errors to a minimum. The need for strict fixation was emphasised. The participants were then given a short practice session until they fully understood the task and were also capable of holding constant fixation. During this time, the eye movements of the participants were carefully watched by the experimenter to ensure that fixation was indeed being held on the fixation point. If the participants' eyes moved from the fixation point, the participants were told of this and reminded of the need for steady fixation. In practice, all participants had little trouble in maintaining fixation. The main experiment was then run. 
For all conditions, each trial commenced with the presentation of the fixation point. After $1000 \mathrm{~ms}$, the cue was presented (see figure 1) upon an otherwise blank (mean luminance) screen. After a further period of $150 \mathrm{~ms}$, the test stimulus was presented. The consistent timings between these events could lead to expectancy effects; however, the use of a choice-discrimination RT mitigates against this problem, as there can be no expectancy as to which button will have to be pressed. Furthermore, any expectancy effects of target onset will be the same under all cueing and target conditions.

The observer made a two-option forced-choice response via a key press whether the target was tilted clockwise or anticlockwise. The participants pressed the ' $<$ ' key for anticlockwise targets or the ' $>$ ' key for clockwise targets. They did so using the first and index finger of their preferred hand. The response caused all stimuli to be removed leaving only the background luminance. The reaction time and response were recorded on the computer for later analysis. The next trial commenced $0.5 \mathrm{~s}$ after this response.

One of four possible targets was presented on each trial, consisting of clockwise or anticlockwise tilt at either high or low contrast. Trials of various cue position, target position, and target contrast were all randomly interleaved within a block of trials so that each of these factors was unpredictable from trial to trial. There were a total of 160 trials in each block. Thus each RT for each participant is based on approximately 40 trials. Each block took approximately 6-7 min. Valid trials are those in which the target location was on the same side as the cue, invalid trials are those on which the target was presented on the opposite side to the cue. Note that the cue is not predictive of the location of the target-it is therefore a purely exogenous cue. It is also not predictive of the response required.

2.1.5 Design and data analysis. Reaction times (RTs) from each trial were collated so as to form four conditions consisting of two levels of cue-target validity (valid versus invalid) and two levels of target contrast (high versus low).

We used a choice-discrimination RT task rather than a simple RT task for the following reasons. First, simple RT tasks require that catch trials are also included to ensure that responding is due to the detection of the target rather than anticipating its presence. Second, it is believed (Sagi and Julesz 1985) that the discrimination of a target may require more attentional resources than the mere detection of its presence. This predicts a greater cueing effect for the choice-discrimination RT task than the simple RT task, allowing a more sensitive test of the experimental manipulations used in the present experiments. In previous experiments we have confirmed this expectancy (Brawn and Snowden 2000; Willey et al, submitted). Third, in experiment 2 we aimed to manipulate the difficulty of this discrimination.

After visual examination of the overall RT data, we decided to treat RTs greater than $1500 \mathrm{~ms}$ or less than $300 \mathrm{~ms}$ as outliers - both were eliminated from the analysis. In both experiments, less than $1 \%$ of trials were eliminated on the slow criterion and less than $2 \%$ on the fast criterion. The small number of these meant that no further analysis was possible. Trials on which the participant made an error were also removed and analysed separately. Any participant who exhibited over $30 \%$ errors in any condition was excluded from any further analysis. Owing to the skewed nature of RTs the median RT was calculated for each condition and for each participant. A visual inspection of the frequency versus RT plots showed the data for each condition to approximate the normal distribution without any major variations in variance. Therefore the data were analysed by using parametric statistics.

\subsection{Results}

One participant was excluded owing to excessive errors. The mean RTs are shown in figure 2. ANOVA showed a main effect of target contrast $\left(F_{1,48}=232.55, p<0.0001\right)$ and of cue validity $\left(F_{1,48}=112.86, p<0.0001\right)$. These main effects were modified by 


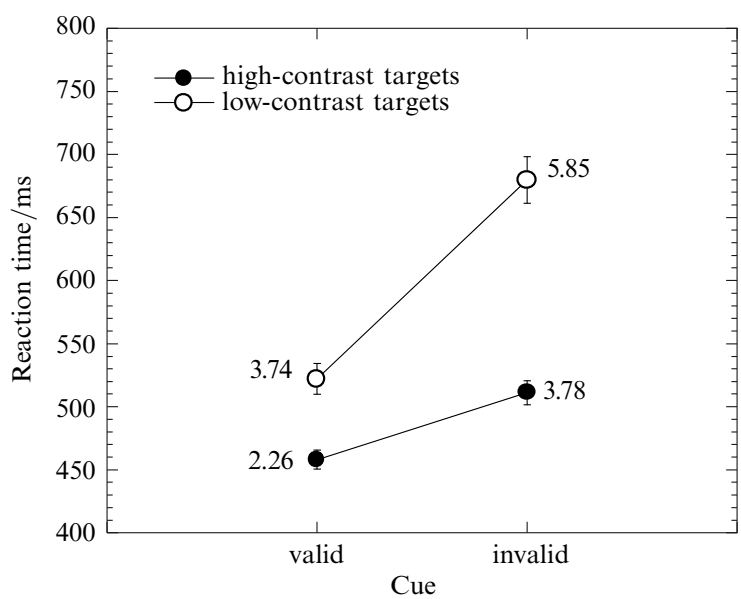

Figure 2. Mean reaction time as a function of cue validity for both highcontrast and low-contrast targets. The numbers next to each symbol are the percentage of errors for each trial type. Error bars represent \pm 1 standard error of the mean.

a significant interaction between the two $\left(F_{1,48}=39.57, p<0.0001\right)$. The interaction is due to a far greater effect of cue validity for the low-contrast targets $(157 \mathrm{~ms})$ than for the high-contrast targets $(58 \mathrm{~ms})$.

A similar analysis of the error rates (see inserts in figure 2) showed a main effect of target contrast $\left(F_{1,48}=20.03, p<0.0001\right)$ and of cue validity $\left(F_{1,48}=23.04, p<0.0001\right)$, but no interaction between the two $(F<1)$.

Hence a greater effect of exogenous spatial cueing as a function of decreasing target contrast was obtained.

\section{Experiment 2: Effect of discrimination difficulty}

In this experiment, all targets were of high contrast, but the tilt of the line from vertical could be small (making the discrimination difficult) or large (see figure 1).

\subsection{Methods}

All methods were identical to those in experiment 1 , save that the targets could be tilted either at $\pm 45^{\circ}$ (easy) or at $\pm 10^{\circ}$ (hard). These orientations were chosen after extensive pilot experiments. The $90^{\circ}$ discrimination is the easiest available, whilst the $20^{\circ}$ one produces a strong effect on RTs whilst still producing a level of errors that we found acceptable. We also found in the pilot studies that the manipulation in contrast (experiment 1) and this level of manipulation of task difficulty produced overall RTs that were similar, making comparisons between the two experiments more plausible. All targets had high contrast. Once again the task of the participant was to say whether the target was tilted clockwise or anticlockwise from the vertical.

The participants were the same as in experiment 1 . Indeed, this experiment was performed in the same session as experiment 1 . The order of experiments was randomised from participant to participant. Again, visual inspection of the data suggested that they approximated a normal distribution for each condition without any gross change in variance.

\subsection{Results}

Four participants were excluded owing to excessive errors. The mean RTs are shown in figure 3. ANOVA showed a main effect of task difficulty $\left(F_{1,45}=220.19, p<0.0001\right)$ and of cue validity $\left(F_{1,45}=102.14, p<0.0001\right)$. These main effects were modified by a significant interaction between the two $\left(F_{1,45}=7.92, p=0.0072\right)$. The interaction is due to a somewhat larger effect of cue validity for the difficult discrimination (74 ms) than for the easy discrimination $(54 \mathrm{~ms})$. 


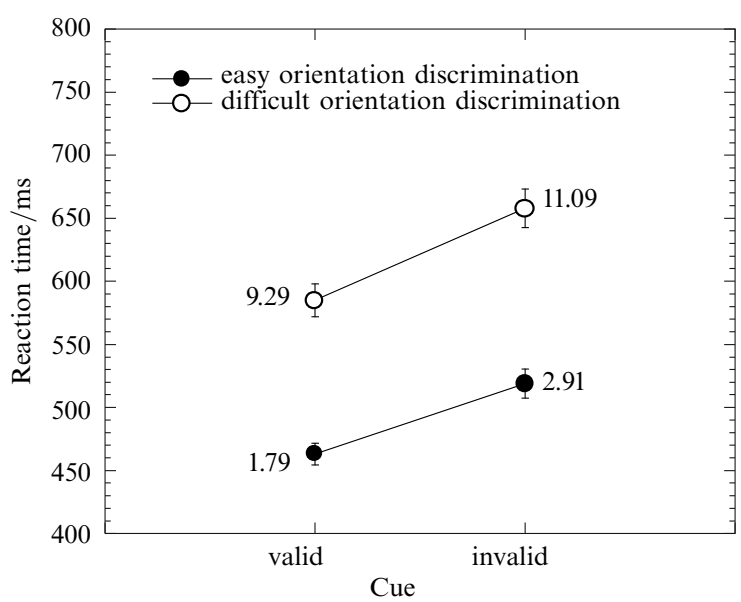

Figure 3. Mean reaction time as a function of cue validity for both easy and difficult orientation discriminations. The numbers next to each symbol are the percentage of errors for each trial type. Error bars represent \pm 1 standard error of the mean.

A similar analysis of the error rates (see inserts in figure 2) showed a main effect of task difficulty $\left(F_{1,45}=60.03, p<0.0001\right)$ and of cue validity $\left(F_{1,45}=8.45, p=0.0056\right)$, but no interaction between the two $(F<1)$.

Hence we find a slightly greater effect of exogenous spatial cueing as the task faced by the subject becomes more difficult.

\section{Comparison of experiments 1 and 2}

We wished to make some simple comparisons between experiments 1 and 2 to ensure that the manipulations of target contrast and task difficulty produced similar changes in RT, and to compare how this manipulation affected the size of the cueing effect. To this end a 3-way ANOVA was performed with cue (valid versus invalid), task level (high versus low - translating to high versus low contrast for experiment 1 and easy versus hard for experiment 2$)$, and experiment $(1=$ target contrast versus $2=$ task difficulty). The first two factors were within-subjects variables. The last was treated as a between-subjects variable because of the exclusion of some participants owing to excessive errors. A similar analysis treating this as a within-subjects variable and excluding any participant who had excessive errors on any particular condition in either experiment produced highly similar results.

For brevity, we present only the crucial comparisons. First, there was no overall difference in RT between experiments $1(544 \mathrm{~ms})$ and $2(557 \mathrm{~ms})(F<1)$, nor was there any interaction between the experiments and level manipulation $\left(F_{1,93}=1.40\right.$ $p>0.1)$. Thus the manipulations in the two experiments appear to have had similar effects on overall RTs as was desired. Second, we wished to examine if the targetcontrast manipulation (experiment 1) had a greater effect on the cueing effect than had the task-difficulty manipulation (experiment 2). This would be seen by a significant 3-way interaction between cue, task level, and experiment. This was indeed significant $\left(F_{1,93}=19.80 p<0.0001\right)$. We conclude that the two manipulations had similar overall effects on RTs, but the size of the cueing effect was increased more by the manipulation of target contrast $(99 \mathrm{~ms})$ than by task difficulty $(20 \mathrm{~ms})$.

\section{General discussion}

In two experiments we found that changes in target contrast produced large changes in the size of the cueing effect for a purely exogenous cue, whilst changes in the difficulty of the task produced only small changes in the cueing effect. 
Our first aim was to contribute to the literature whether luminance or contrast of the target affects the cueing effect, given the contradictory pattern of results reported previously. We obtained a strong interaction such that the cueing effect approximately trebled when we compared low-contrast versus high-contrast targets. The negative results of Hughes (1984) have been explained by the blocking of trials that may have allowed different strategies for the different targets (Hawkins et al 1988). However, this criticism does not apply to the study of Tellinghuisen et al (1996), as they interleaved the high-contrast and low-contrast targets, yet did not find an interaction. Thus at this moment the negative results, particularly those of Tellinghuisen et al (1996), are contradictory to the ones reported here. The only obvious difference between their experiment and our own is that Tellinghuisen et al used an endogenous cue (one that is symbolic with respect to the likely location of the forthcoming target) whereas we used an exogenous cue (one which attracts attention to its own location but gives no indication where the target will appear). Clearly further studies are needed to investigate this issue.

Where might the effects of target contrast upon the cueing effect reside? We have argued that contrast should affect both the movement of attention and the processing of a target; hence an interaction of contrast and cue does not reveal which of these processes is responsible for the cueing effect. To examine this issue we manipulated the similarity between possible targets that the participant must discriminate. Such a change should not alter the 'salience' of the target against its background and therefore should not interact with the signal to move attention on the invalid trials. Thus any interaction with this manipulation should be due to the processing stage. Our results did show a significant interaction, though the effect is small. It is important to note that these effects of target contrast and task difficulty both altered the overall RTs by approximately the same amount. Hence one cannot explain the difference in the cueing effects by what one might expect from a mere slowing of RTs.

It has been shown that an observer's expectancy of the target's properties can moderate the capture of attention (Folk et al 1992; Gibson and Kelsey 1998). Could such 'higher-level' processes account for the results we present? This seems most unlikely. Crucially within each experiment the two target levels (contrast in experiment 1 and difficulty in experiment 2) were interleaved within a block of trials. Hence the observer has the same expectancy about the target for both low and high levels of contrast. Thus differences in expectancy can not be used to explain the difference in the validity effect as a function of contrast.

Taken together, the results from the two experiments suggest that target contrast has its major effect upon validity effects at an early stage of processing (Johannes et al 1995), and is consistent with a reduced ability of the target to attract attention to itself for further processing. As such, it is important that future research takes into account target salience, and how factors such as old age, disease, etc might alter target salience, before we can make conclusions about attentional mechanisms.

Acknowledgements. This work was supported by a grant from the Wellcome Trust and a postgraduate studentship to Jennifer Willey jointly funded by the Caswell Clinic, Glanrhyd Hospital, Bridgend, and the School of Psychology, Cardiff University. Thanks also to Anna Jones, Marc Isaacs, and Angela McCarthy for help with data collection.

\section{References}

Bennett K M B, Waterman C, Scarpa M, Castiello U, 1995 "Covert visuospatial attentional mechanisms in Parkinson's disease" Brain 118 153-166

Bonnel A-M, Stein J-F, Bertucci P, 1992 "Does attention modulate the perception of luminance changes?" Quarterly Journal of Experimental Psychology A 44601 -626 
Brawn P T, Snowden R J, 2000 "Attention to overlapping objects: detection vs discrimination of luminance changes" Journal of Experimental Psychology: Human Perception and Performance $26342-358$

Bundesen C, 1990 "A theory of visual attention" Psychological Review $97523-547$

Corbetta M, Miezin F M, Shulman G L, Petersen S E, 1993 "A PET study of visuospatial attention" Journal of Neuroscience $131202-1256$

Downing C J, 1988 "Expectancy and visual spatial-attention: effects on perceptual quality" Journal of Experimental Psychology: Human Perception and Performance 14 188-202

Enns J T, Brodeur D A, 1989 "A developmental study of covert orienting to peripheral visual cues" Journal of Experimental Child Psychology 48171 - 189

Folk C L, Remington R W, Johnston J C, 1992 "Involuntary covert orienting is contingent on attentional control settings" Journal of Experimental Psychology: Human Perception and Performance 181030 - 1044

Gibson B S, Kelsey E M, 1998 "Stimulus-driven attentional capture is contingent on attentional set for displaywide visual features" Journal of Experimental Psychology: Human Perception and Performance $24699-706$

Hawkins H L, Shafto M G, Richardson K, 1988 "Effects of target luminance and cue validity in the latency of visual detection" Perception \& Psychophysics 44 484-492

Hughes H C, 1984 "Effects of flash luminance and positional expectancies on visual response latency" Perception \& Psychophysics $36177-184$

Johannes S, Münte T F, Heinze H J, Mangun G R, 1995 "Luminance and spatial attention effects on early visual processing" Cognitive Brain Research 2 189-205

LaBerge D, Brown V, 1989 "Theory of attentional operations in shape identification" Psychological Review $96101-124$

Maruff P, Pantelis C, 1999 "Attention and neuropsychiatric disorders" Current Opinion in Psychiatry $12339-344$

Müller H J, Rabbit P M A, 1989 "Reflexive orienting of visual attention: time course of activation and resistance to interruption" Journal of Experimental Psychology: Human Perception and Performance 15315 - 330

Nissen M J, Corkin S, 1985 "Effectiveness of attentional cueing in older and younger adults" Journal of Gerontology $\mathbf{4 0} 185-191$

Parasuraman R, Greenwood P M, Haxby J V, Grady C L, 1992 "Visuospatial attention in dementia of the Alzheimer type" Brain $115711-733$

Posner M, Petersen S E, 1990 "The attention system of the human brain" Annual Review of Neuroscience $1325-42$

Posner M I, Raichle M E, 1997 Images of Mind Scientific American Library

Posner M I, Snyder C R R, Davidson B J, 1980 "Attention and the detection of signals" Journal of Experimental Psychology: General 109 160-174

Posner M I, Walker J A, Friedrich F J, Rafal R D, 1984 "Effects of parietal injury on covert orienting of attention" Journal of Neuroscience 41863 - 1874

Rafal R D, Robertson L C, 1995 "The neurology of visual attention", in The Cognitive Neurosciences Ed. M Gazzaniga (Cambridge, MA: MIT Press) pp 625-648

Riggio L, Kirsner K, 1997 "The relationship between central and peripheral cues in covert visual orientation" Perception \& Psychophysics $59885-899$

Sagi D, Julesz B, 1985 “'Where' and 'what' in vision” Science $2281217-1219$

Sclar G, Maunsell J H R, Lennie P, 1990 "Coding of image contrast in central visual pathways of the macaque monkey" Vision Research $301-10$

Shapley R, 1986 "The importance of contrast for the activity of single neurons, the VEP and perception" Vision Research 26 45-61

Theeuwes J, 1994 "Exogenous and endogenous control of visual selection" Perception 23 429-440

Tellinghuisen D J, Zimba L D, Robin D A, 1996 "Endogenous visuospatial precueing effects as a function of age and task demands" Perception \& Psychophysics 38 947-958

Willey J, Gray N S, Snowden R J (submitted) "Attentional cueing in schizophrenic patients: validity and alerting effects" 
